\title{
Oral Biopsy in Dental Practice - The Pathologist's Perspective
}

Khoo SP. Oral Biopsy in Dental Practice - the Pathologist's Perspective.

Annals Dent. Univ. Malaya 1995;2:29-32.

\begin{abstract}
ABSBRACT
The ability of a pathologist to accurately interpret a lesion is largely dependent on a good biopsy performed by the surgeon. This article describes the common piffalls in biopsy techniques and of ers ways to minimize them. It also discusses the difficulties encountered by the pathologist in attempting to interpret artefactually damaged tissues.
\end{abstract}

Key Words: Biopsy, Dental, Pathologist.

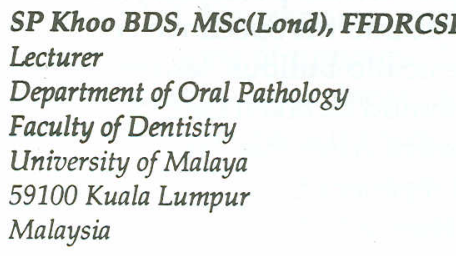

\section{INTRODUCTION}

Biopsy is defined as "the removal and excision of tissue or other material from the living body for purposes of diagnosis"(1). It remains the most reliable check on the clinical diagnosis. There are basically two reasons for performing a biopsy: (a) to establish a definitive diagnosis as early as possible so that correct treatment may be initiated without delay and (b) to determine whether an abnormality has been completely removed.

The accuracy of the diagnosis and subsequently the treatment are dependent upon the surgeon's ability to biopsy adequate and representative lesional tissue, sufficient fixation of the tissue and the oral pathologists ability to interpret the histologic sections. Therefore the tissue provided, for whatever reason is unsuitable, the biopsy is considered to have failed.

The Department of Oral Pathology, Dental Faculty, University of Malaya receives an increasing number of biopsies each year, with those from the practitioners outside the faculty making up one-third of the total. Out of 3121 specimens received 188 were undiagnostic (Table 1). The main reasons for non-specificity or being non-diagnostic are summarized in Table 2. A survey carried out at the Division of Stomatology, Institute for Medical Research, Kuala Lumpur showed that 17\% of the oral pathology specimens were nonspecific(2). This volume of non-diagnosffc biopsies can be reduced as they are of no value to either the clinician nor the patient.

The aims of this paper are to:

(1) outline the common pitfalls in biopsy techniques

(2) discuss difficulties encountered by the oral pathologist in attempting to interpret artifactually damaged tissues and

(3) discuss ways to minimse or eliminate these alterations.

Specimens removed from the oral cavity are often small and the possibility of producing artifacts is thus enhanced. The common pitfalls are discussed in three categories ie. pitfalls before biopsy, during biopsy and after biopsy.

\section{PITFALLS BEFORE BIOPSY}

There is little which can be done to prevent manipulation of a lesion by misinformed patients before a biopsy is taken such as, the application of various medications to a lesion or tissue biting. On the part of the surgeon, he should not inject local anaesthetic solution directly into the biopsy site but around the periphery instead. Direct infiltration of anaesthetic solution will cause volumetric distortion of the relationship of the tissue in the biopsy specimens.

\section{PITFALLS DURING THE BIOPSY PROCEDURE}

Most tissue alterations which occur during this stage are avoidable and are discussed below:

\section{(1) Tissue selection}

When performing an incisional biopsy, the most representative portions of the lesion should be selected to enable the pathologist to examine the maximum volume of abnormal tissue. In addition, a lesion with varying appearances requires more than one biopsy and the site of maximal clinical activity should be included. Biopsies of epithelium only are usually nondiagnostic, or at best, only partially diagnostic, because the nature or any associated connective-tissue changes cannot be assessed histologically (Fig. 1)

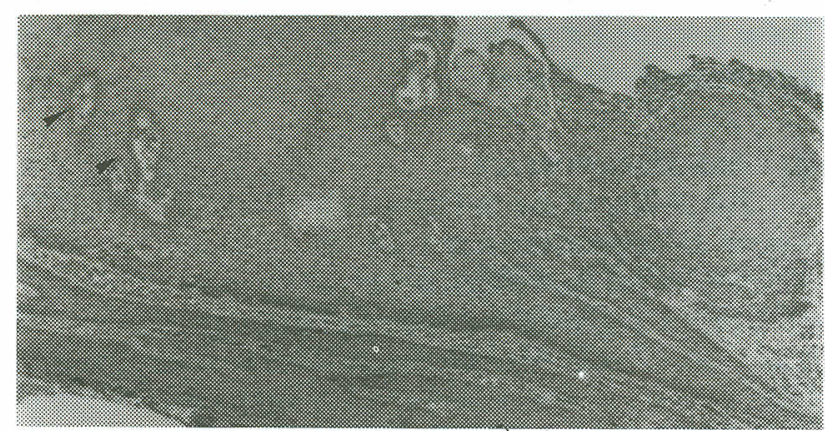

Figure 1. Section from a biopsy specimen mainly epithelium. Connective tissue is seen as islands within epithelium (arrow). Histopathologic 'section of lesions such as these are inadequate and difficult to interpret. ( $H \& E$, magnification X 20). 
For an incisional biopsy of an ulcer or any condition involving sloughing mucosa, it is important to include a margin of normal tissue. A simple means of accomplishing this is to pass a length of suture through the sloughing portion into submucosa and then out through normal' adjacent mucosa. The length of suture facilitates atraumatic handling of the delicate specimen and will prevent the potentially sloughing mucosa from floating free into the specimen container during transit.

In vesicullo-bullous lesions, as early a lesion as possible should be taken for biopsy, preferably not older than 48 hours. After this period a signifficant amount of epithelial regeneration will have taken place at the floor of the blister which may dislocate the bulla from its primary site. With the exception of large bullous lesions, an excisional biopsy should be carried out including the edge of a new lesion extending into normal mucosa or skin. A biopsy taken at the edge of the ulcerated bulla may show only denuded epithelium (Fig. 2) so that even immunofluorescence may be useless.

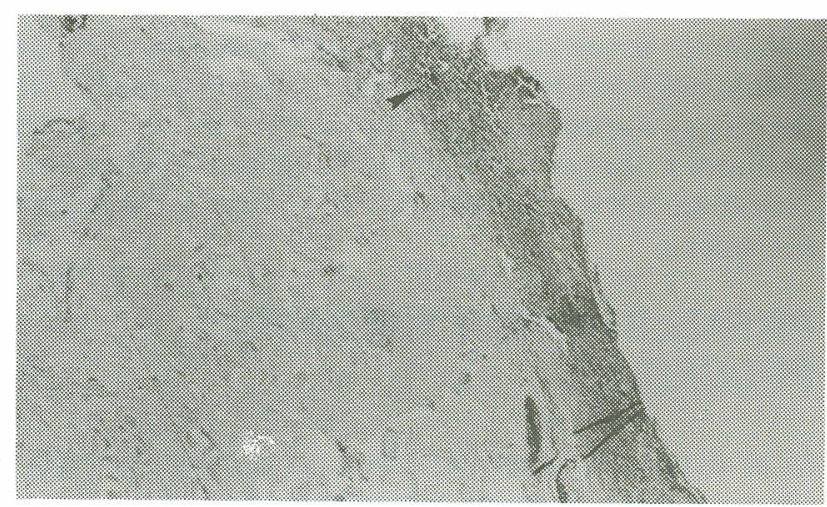

Figure 2. An incisional biopsy of a vesicullo-bullous lesion on the palate exhibiting only connective tissue. A moderate degree of inflammation is present within lamina propria (arrow). Interpretation is impossible without the overlying epithelium. ( $H \& E$, magnification $X 40$ ).

Biopsy of tumours are of particular importance. A friable area, which on histology often shows necrotic tissue, should not be selected for biopsy as it is of limited value. A biopsy specimen of adequate depth and breadth will enable the oral pathologist to assess the tumour more accurately, such as its stages or depth of invasion, the tumour-host relationship and its pattern of spread. These features have been shown to correlate better with prognosis of the disease $(3,4)$.

\section{(2) Insufficient tissue}

The ability of the pathologist to correctly interpret a biopsy is dependent not only on the quality but the quantity of the specimen(5). Small biopsies are not only difficult to orientate correctly but run a greater risk of being lost in handling. Shrinkage occurs during fixation and processing, thus further reducing the usefulness of a tiny biopsy. Biopsies should be about $1.0 \times 0.5 \times$ $0.5 \mathrm{~cm}$.

The ideal shape of a mucosal biopsy is either elliptical or round as either shape provides satisfactory volumes of surface area. The former is preferred when using a scalpel.

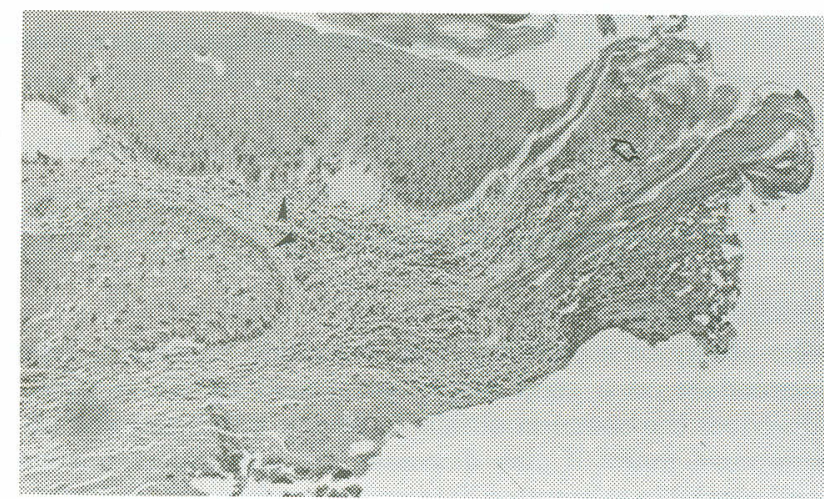

Figure 3. A biopsy of mucous membrane exhibiting laser-induced artefacts (white arrow). A diagnosis of squamous cell carcinoma was made basing upon the histomorphology of adjacent, less coagulated areas (black arrow). Assessment of the presence of carcinoma at this excision margin was impossible because of electrosurgically induced artefact. ( $H \& E$, magnification $X 40$ )

\section{(3) Use of electrosurgery and lasers}

Electrosurgical blades or lasers can produce artefacts (Fig. 3) which can interfere with microscopic evaluation especially of dysplastic or malignant lesions(5). Electrosurgically, induced wounds have been shown to heal more slowly than scalpel induced wounds(6). With this added disadvantage, the use of electrosurgery or lasers for obtaining biopsies is contraindicated except perhaps in instances where evaluation of tissue margins is not necessary or in patients where haemostasis is a signifficant problem. Even then in the case of the latter, bladed instruments can be used for initial removal of the specimen and bleeding points of the surgical bed subsequen fly treated by electrocoagulation(6).

\section{(4) Tissue manipulation artefacts}

Biopsies of the buccal mucosa, lips, floor of mouth and tongue are often to perform because the tissue retreats as the scalpel is applied. To overcome this some surgeons firmly grasp the lesion with forceps or haemostats, pulling the tissue and incising below the instrument. This makes histologic interpretation difficult or sometimes impossible.

Toothed forceps when applied too forcefully, leave puncture holes often resembling mucosal pits, epidermoid cysts or even sinus-like intrusion histologically. Lymph nodes, especially, should be handled with extreme care as the relationship of the whole architecture of the Iymph nodes is vital for interpretation. To overcome this, placement of a length of suture through the biopsy specimen affords control and atraumatic handling. It also helps prevent aspiration into the sucker.lf forceps are used, they should be applied gently to an area of normal tissue included in the biopsy, and never onto lesional tissue.

Thin specimens often curl up when fixed. This is undesirable because the orientation between connective tissue and epithelium will usually be lost, especially if the tissue does not posses an identifiable submucosa or muscle base. Interpretation is made easier when specimens have been fixed flat. This can be achieved by spreading out the specimen on a piece of blotting or filter paper and gently lowering into the fixative. The specimen generally adheres to the paper for sufficiently 
long to remain flat during fixation. Alternatively, noncorrosive pins can be used to secure the specimen to a piece of rigid cardboard.

\section{(5) Unnecessary additions}

Occasionally unwanted tissues or materials are inadvertently included in the biopsy specimen, rendering histologic interpretation needlessly difficult. This is especially true for gingival abscesses which include a fragment of calculus or pbque which may histologically mimic a local actinomycotic infection. Contaminants by numerous starch granules from surgical gloves can suggest an erroneous aetiology to a tissue section of a foreign body granuloma. Other unwanted additions include tissue dyes (eg. gentian violet to orientate a specimen).

\section{POST-BIOPSY PROCEDURES}

\section{(1) Fixation problems}

When a biopsy specimen is removed, it should immediably be immersed in a fixative solution eg. $10 \%$ buffered formalin. Fixation is required to arrest autolysis and putrefaction and to stabilise the protein of the cells. The specimen should not be left on the counter top or tray where it will dehydrate and autolyse(Fig. 4). Ideally, the total volume of the fixing solution should be at least 20 times the size of the tissue specimen(7). There have been occasions where specimens arrive at the laboratory, sitting on the container wall way above the level of the formalin, occasionally bathed by the fixative during transit. These specimens are as bad as dried up ones.

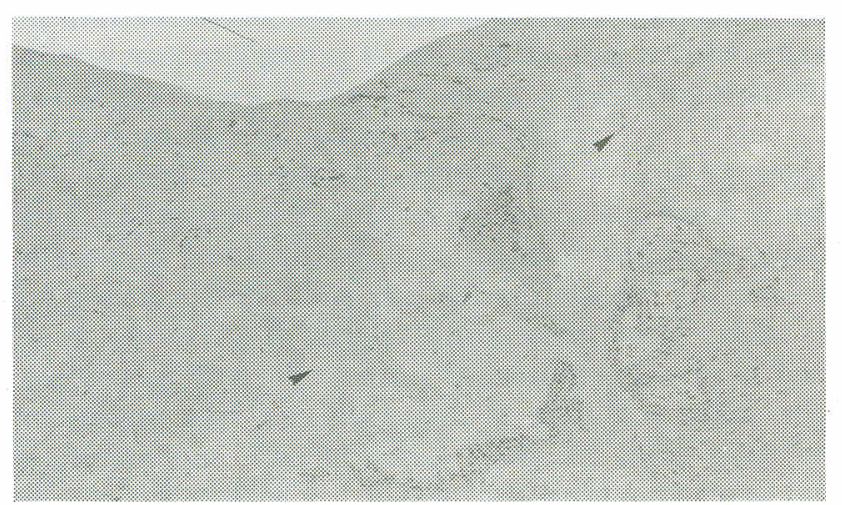

Figure 4. Section of a biopsy specimen not fixed in formalin for two days. Autolysis has taken place and most of the areas show an acellular appearance (arrows) due to widespread cellular degeneration. The difficulty in interpretation is obvious ( $H \& E$, magnification $\mathrm{X} 40$ )

A good fixative will penetrate tissue quickly, will preserve cellular detail and will harden the specimen to protect it from the various stages of tissue processing in the laboratory. Currently, $10 \%$ neutral buffered formalin is a highly regarded fixative.

Water and saline are unsuitable transport media for biopsied tissues. Specimens should not be rinsed with water before fixing nor should it be put into saline or water first and transferred later into formalin. Specimens placed in water or saline or buffered solution (ie. when formalin in the container has evaporated leaving behind white powder to which one adds water) produce artefacts resembling pemphigus vulgaris histologically due to autolysis(8). The effects of using different fixatives are referred to by Thompson and coworkers(9).

\section{(2) Specimen orientation}

The use of sutures to tag important landmarks is of considerable help to the pathologist and will markedly enhance the accuracy of his reports, particularly regarding clearance of margins of tumours. Tagging of sites should be accompanied by an explanatory diagram for it to be meaningful. This is especially more important in small excisions without any identifiable landmarks.

The specimens sent in should be accompanied by a form containing relevant data including the patient's name, age, sex, race, chief complaint, duration of complaint, clinical data, provisional and differential diagnoses. This should be written in legible handwriting with ninimal use of known abbreviations. Bony lesions often require further information for accurate diagnosis, such as radiological and biochemical findings.

Specimen bottles should be handled with care to prevent damage during trainsit. Clear plastic or glass bottles are commonly used but not test tubes with cotton plugs. The specimen bottles should be securely closed to prevent evaporation of fixative solution or unnecessary spillage.

\section{(3) Surgeon-pathologist relationship}

The interdependence between the clinician/surgeon and the pathologist is of fundamental importance. A free communication between these two disciplines is vital to clear any doubts that may arise in interpreting the biopsy specimen taken by the surgeon or in the diagnosis provided by the pathologist.

Specimens should ideally be sent to one pathologist for any particular case rather than splitting up the specimen. This is because different areas within a specimen may show different histological pattern. Such is true of cases often encountered in diagnosis of salivary gland tumours or some odontogenic tumours. Different diagnoses may be produced as a result.

The request of the pathologist for a second biopsy is sometimes required where there is any doubt regarding the adequacy or representative nature of the original specimen. This can be minimized if the avoidable pitfalls mentioned above are considered carefully when performing a biopsy.

\section{CONCLUSION}

The proper preparation of a tissue for microscopic analysis is dependent on steps taken by the surgeon, assistant and technician to reduce the inclusion of artefacts. This article has attempted to discuss and Illustrate some significant artifactual alterations and ways to minimse or eliminate them. The suggestions offered here will help dental surgeons to obtain tissue of excellent quality for the oral pathologist to make accurate diagnostic interpretation for the benefit of the patient. 


\section{REFERENCES}

1. Joblonski S. In Illustrated Dictionary of Dentistry. WB Saunders Company, 1982: p104.

2. Ramanathan K, Chelvanayagam Pl. The Biopsy: Simple aids to quick and correct histopathological diagnosis. Dent J Malaysia 1983 $6: 105-108$.

3. Crissman JD, Lin WY, Gluckman JL, Cummings G. Prognostic value of histopathologic parameter in squamous cell carcinoma of the oropharynx. Cancer 1984;54:2995-3001.

4. Anneroth G, Batsakis J, Luna M. Review of the literature and a recommended system of malignancy grading in oral squamous cell carcinomas. Scan J Dent Res 1987, 95:229-49.

5. Zegarelli DJ. Common problems in biopsy procedures. J Oral Surg 1978; 36:644-47.

6. Sozio RB, Riley EJ, Shklar G. A controlled study of electrosurgical current and wound healing. Oral Surg 1976;41:709-17.

7. Sheehan DC, Hrapchak BB. Theory and practice of histotechnology, 2nd edn. St. Louis, CV Mosby, 1980.

8. Weir JC, Weathers DR. A fixation artefact simulating acantholytic disease. Oral Surg 1976;41:105-8.

9. Thompson SW, Luna LG. An atlas of artefacts encountered in the preparation of microscopic tissue sections. Springfield edn. Charles C Thomas, 1978. 